\title{
A Combined Method in Parameters Optimization of Hydrocyclone
}

\author{
Jing-an Feng, ${ }^{1,2}$ Xiao-qi Tang, ${ }^{1}$ Wei-bing Wang, ${ }^{2}$ Rui Ying, ${ }^{2}$ and Ting Zhang ${ }^{2}$ \\ ${ }^{1}$ School of Mechanical Science and Engineering, Huazhong University of Science and Technology, Wuhan 430074, China \\ ${ }^{2}$ College of Mechanical and Electrical Engineering, Shihezi University, Shihezi 832003, China \\ Correspondence should be addressed to Wei-bing Wang; wwb_mac@shzu.edu.cn
}

Received 17 August 2016; Accepted 25 October 2016

Academic Editor: Sandro Longo

Copyright (c) 2016 Jing-an Feng et al. This is an open access article distributed under the Creative Commons Attribution License, which permits unrestricted use, distribution, and reproduction in any medium, provided the original work is properly cited.

\begin{abstract}
To achieve efficient separation of calcium hydroxide and impurities in carbide slag by using hydrocyclone, the physical granularity property of carbide slag, hydrocyclone operation parameters for slurry concentration, and the slurry velocity inlet are designed to be optimized. The optimization methods are combined with the Design of Experiment (DOE) method and the Computational Fluid Dynamics (CFD) method. Based on Design Expert software, the central composite design (CCD) with three factors and five levels amounting to five groups of 20 test responses was constructed, and the experiments were performed by numerical simulation software FLUENT. Through the analysis of variance deduced from numerical simulation experiment results, the regression equations of pressure drop, overflow concentration, purity, and separation efficiencies of two solid phases were, respectively, obtained. The influences of factors were analyzed by the responses, respectively. Finally, optimized results were obtained by the multiobjective optimization method through the Design Expert software. Based on the optimized conditions, the validation test by numerical simulation and separation experiment were separately proceeded. The results proved that the combined method could be efficiently used in studying the hydrocyclone and it has a good performance in application engineering.
\end{abstract}

\section{Introduction}

Carbide slag is an industrial waste yielded by calcium carbide hydrolysis in the process of producing $\mathrm{C}_{2} \mathrm{H}_{2}$ gas and PVC [1]. Millions of tons of carbide slag accumulate, leading to many environmental problems [2]. Accordingly, comprehensive utilization of carbide slag emerges to be an urgent issue. The main component of carbide slag is $\mathrm{Ca}(\mathrm{OH})_{2}$, as well as some elements of impurity phase such as $\mathrm{Fe}, \mathrm{Si}, \mathrm{Al}$, and $\mathrm{Mg}[1-3]$. The key to achieve the resource utilization of carbide slag is effective removal of the impurities in it.

Hydrocyclones are widely utilized in mineral industries for classification and separation of solid particles of different sizes and densities suspended in water medium $[4,5]$. It is applied to a series of separating processes such as solidsolid, solid-liquid, liquid-liquid, and solid-gas, which are treated frequently in many industrial fields such as mining, chemistry, petrol, biology, and medicine [6]. Since the elements phase of the impurities and $\mathrm{Ca}(\mathrm{OH})_{2}$ differences in their density, through the carbide slag grinded into a certain particle size, carbide slag particles which contain $\mathrm{Ca}(\mathrm{OH})_{2}$ and impurities can be dispersed. Based on the density difference between $\mathrm{Ca}(\mathrm{OH})_{2}$ and impurities, there is the possibility for the carbide slag impurities to be removed by the hydrocyclone.

High-performance hydrocyclone depends on the best match among physical parameters, structural parameters, and operating parameters, that is, the optimization of key parameters which influence the motion behaviors of solid particles and their separation results [6]. Although there have been a few of empirical and theoretical models to selection for hydrocyclone, it is still impossible to have one model which can generally use hydrocyclones satisfactorily [7-10]. The experimental method is still the common method for parameters optimization in particular system, but it is usually through the single factor analysis to acquire the better result. For example, on study of the separation performance of air-injected de-oil hydrocyclones, Zhao et al. [11] used the experimental method to optimize the effects of different 
geometric parameters of hydrocyclone, the method does not consider the interaction effect among the various parameters of hydrocyclone, so the parameters selected cannot be the optimum.

DOE (Design of Experiment) is a kind of statistical method used in experimental arrangements and experimental data analysis. With the small-scale test, shorter test cycles and lower test costs achieve the desired results and draw scientific conclusions; the DOE method has been widely used in optimization area [12-15]. Through the central composite design method, Arokiadass et al. [16] developed a mathematical model for tool flank wear correlation with the spindle speed, feed rate, depth of cut, and various mass fraction of SiCp and obtained the optimal process parameter combination with the model. Similarly, the method is also used in optimization of hydrocyclone. In order to produce bentonite concentrate with hydrocyclone, Özgen et al. [4] used a three-level Box-Behnken factorial design combined with the response surface methodology for modeling and optimizing. The experimental variables include the operation parameters and structure parameters of the hydrocyclone with feed solid, inlet pressure, diameter of the apex, and diameter of the vortex. Simultaneously, the method can be also used to arrange the experiment combined with the other technology in optimization [17]. Clearly, the experimental design method has good effectiveness to the optimization for the small number of tests and lesser test time and costs; however, due to the large amount of the impact factors, especially the structure factors involved in the hydrocyclone, only a few key factors can be selected and be optimized, and, also due to the amount of experimental works and because it costs too much, the DOE method is restricted.

More recently, the advent of high speed computational systems made researchers focus on performance simulations using Computational Fluid Dynamics (CFD) techniques [1823]. The numerical simulation based on CFD has become an effective approach to investigating the dynamical characteristics of flow and particles, which have been validated in accord with the experimental data nearly. He et al. [24] applied the numerical simulation method that simulated the efficiency for particles with low concentration in a hydrocyclone. The result showed the modified $k-\varepsilon$ model produces results in good agreement with experimental data. Swain and Mohanty [5] used the numerical simulation method with the EulerianEulerian model that simulated a solid-liquid hydrocyclone with two solid phases and one liquid phase in the model being taken into account. The result showed that the separation efficiency predicted by $k-\varepsilon$ model is close to that predicted by the RSM model for the low flow rates. Bhaskar et al. [22] compared the simulated results generated using different turbulence models, that is, standard $k-\varepsilon, k-\varepsilon$ RNG, and RSM in terms of water throughput and split with the help of suitably designed experiments. The result showed the predictions using RSM model were better in agreement with experiment which resulted in a marginal error between $4 \%$ and $8 \%$. Wang and $\mathrm{Yu}$ [21] used the RSM model that simulated the particle flow described by the stochastic Lagrangian model. The flow features are examined in terms of the flow field, pressure drop, split ratio, and the model validated by the
TABLE 1: Structural parameters used in simulation.

\begin{tabular}{lc}
\hline Structural parameter & Value \\
\hline Cyclone diameter/mm & 60 \\
Length and width of feed inlet $(L / B) / \mathrm{mm}$ & $16 / 7$ \\
Diameter of overflow pipe/mm & 18 \\
Depth of overflow pipe $/ \mathrm{mm}$ & 45 \\
Height of cylindrical section $/ \mathrm{mm}$ & 70 \\
Cone angle/degree & $15^{\circ}$ \\
Diameter of underflow pipe $/ \mathrm{mm}$ & 12 \\
\hline
\end{tabular}

good agreement between the measured and predicted results. Delgadillo and Rajamani [20] used the numerical simulation method compared $k-\varepsilon$ model, the Reynolds stress model, and the large-eddy simulation model for the predictions of aircore dimension, mass split, and axial and tangential velocities. The result showed that the large-eddy simulation model was clearly closer in predicting the experimental data than the other two. Aforementioned, numerical simulation based on CFD technique has a good agreement in predicting the performance of hydrocyclone with experimental result, and the RSM turbulences model can obtain a good result compared to kinds of $k-\varepsilon$ models but low computing requirements compared to the large-eddy simulation model.

The numerical simulation result could substitute for the actual experiment in some degree if the simulation method was selected properly; the present study combined with the DOE method and the numerical simulation based on the CFD technical to optimize the key parameters of the hydrocyclone achieves efficient separation of calcium hydroxide and impurities in carbide slag.

\section{Experiments}

2.1. Model Descriptions. The structural parameters of the hydrocyclone used are shown in Table 1 [25]. The geometry of the hydrocyclone with $3 \mathrm{D}$ body was created using the commercial software, GAMBIT (a preprocessor for FLUENT), and was divided into four parts, that is, slurry inlet, cylindrical section, cone section, and overflow pipe. The grid of each part was generated in Cooper way with structure mesh. The grid has 197417 cells and has the mesh independence test, and the content of the mesh independence test includes pressure, velocity, and mass flow rate.

The simulation used FLUENT which is a kind of popular and commercial software based on the CFD. FLUENT provides lots of turbulent models and the Reynolds stress model is selected in the present study as it predicts a more accurate solution for complex flows than the standard $k$ - $\varepsilon$ mode [22]. Particularly, the 3D pressure based solver was used with the first-order implicit unsteady-state formulation.

The multiphase model is selected with the mixture model, allowing the high slurry concentration in simulation. The multiphase model consists of three phases which are primaryphase water and secondary-phases, that is, $\mathrm{Ca}(\mathrm{OH})_{2}$ and FeSi. The material type of all phases is defined as fluid. The solid phases, that is, $\mathrm{Ca}(\mathrm{OH})_{2}$ and $\mathrm{FeSi}$, are defined as granular type and have the same granularity in the same group of 
simulation. The secondary-phases have the same Granular Viscosity and the value was calculated by related formulation.

The momentum equations are discretized using the bounded central differencing method. The SIMPLEC method is used for the pressure-velocity coupling. The discretization equation of pressure used "PRESTO!" and the other equations use the second-order upwind.

The iteration of time step size was set at $0.0001 \mathrm{~s}$, the number of time steps was set at 30000, and the convergence criteria through the surface mass flow rate of overflow and underflow reached steady state.

The boundary conditions are set as follows:

(1) Velocity inlet is set at the measured flow velocity magnitude.

(2) Pressure outlet is set at zero, which is expressed with relative pressure.

(3) The no-slipping boundary condition is put on all walls, where every velocity component is zero.

(4) The turbulence specification method of velocity inlet used intensity and hydraulic diameter, and the values are calculated by related formulation; the pressure outlet used $k$ and Epsilon with default value.

(5) The volume fraction of the two solid phases is given in Table 2 and the computational method related to the volume fraction is presented in Table 2 .

Based on the experimental analysis of carbide slag, assume that the mass concentrations of $\mathrm{Ca}(\mathrm{OH})_{2}$ and $\mathrm{FeSi}$ were defined, respectively, as $\omega_{1}=0.85$ and $\omega_{2}=0.15$ and the density was defined as $\rho_{1}=2.234 \mathrm{t} / \mathrm{m}^{3}$ and $\rho_{2}=3.2 \mathrm{t} / \mathrm{m}^{3}$. The minimum density of impurities used FeSi to ensure the applicability of the test results to other impurities. The data of the volume fraction listed in Table 2 were obtained according to the mass concentration and calculated by

$$
\begin{aligned}
\rho & =\frac{\rho_{1} \rho_{2}}{\omega_{1} \rho_{2}+\omega_{2} \rho_{1}}, \\
\phi_{1} & =\frac{\omega_{1} C_{w} \rho}{\rho_{1}\left[\left(1-C_{w}\right) \rho+C_{w}\right]}, \\
\phi_{2} & =\frac{\omega_{2} C_{w} \rho}{\rho_{2}\left[\left(1-C_{w}\right) \rho+C_{w}\right]} .
\end{aligned}
$$

(6) The computational method related to the hydraulic diameter, turbulent intensity, and Granular Viscosity is presented as follows:

$$
\begin{aligned}
I= & 0.16 \operatorname{Re}^{-1 / 8}, \\
d_{H} & =\frac{2 L B}{L+B}, \\
\mu_{m} & =0.001003\left(1+2.5 C_{v}+10.05 C_{v}{ }^{2}\right. \\
& \left.+0.00273 \exp \left(16.6 C_{v}\right)\right),
\end{aligned}
$$

\begin{tabular}{|c|c|c|c|c|c|c|}
\hline \multirow{2}{*}{ Number } & \multirow{2}{*}{ Factor } & \multicolumn{5}{|c|}{ Level } \\
\hline & & -1.682 & -1 & 0 & +1 & +1.682 \\
\hline 1 & $\begin{array}{c}\text { Slurry concentration } \\
\qquad A\left(x_{1}\right)\end{array}$ & 0.03 & 0.10 & 0.20 & 0.30 & 0.37 \\
\hline 2 & $\begin{array}{c}\text { Inlet velocity } \\
B\left(x_{2}\right)(\mathrm{m} / \mathrm{s})\end{array}$ & 1.64 & 3.00 & 5.00 & 7.00 & 8.36 \\
\hline 3 & $\begin{array}{l}\text { Granularity } \\
C\left(x_{3}\right)(\mu \mathrm{m})\end{array}$ & 3.18 & 10.00 & 20.00 & 30.00 & 36.82 \\
\hline
\end{tabular}

TABLE 2: Computed result of volume fraction.

\begin{tabular}{lcc}
\hline $\begin{array}{l}\text { Mass concentration } \\
C_{w}\end{array}$ & $\begin{array}{c}\text { Volume fraction } \\
\text { of } \mathrm{Ca}(\mathrm{OH})_{2} \varphi_{1}\end{array}$ & $\begin{array}{c}\text { Volume fraction } \\
\text { of } \mathrm{FeSi} \varphi_{2}\end{array}$ \\
\hline 0.03 & 0.0116 & 0.0014 \\
0.10 & 0.0404 & 0.0053 \\
0.20 & 0.0859 & 0.0106 \\
0.30 & 0.1378 & 0.0170 \\
0.37 & 0.1786 & 0.0220 \\
\hline
\end{tabular}

TABLE 3: Experimental parameters and their levels.

$$
\begin{aligned}
\operatorname{Re} & =\frac{\rho_{m} v_{i} d_{H}}{\mu_{m}}, \\
\rho_{m} & =\frac{\rho}{C_{w}+\rho\left(1-C_{w}\right)}, \\
C_{v} & =\frac{C_{w}}{\rho+C_{w}(1-\rho)},
\end{aligned}
$$

where $\rho$ is ore density, $\mathrm{kg} / \mathrm{m}^{3}$; I is turbulent Intensity; Re is Reynolds number; $d_{H}$ is hydraulic diameter, $\mathrm{mm} ; C_{v}$ is volume concentration; $\mu_{m}$ is Granular Viscosity, $\mathrm{kg} / \mathrm{ms} ; \rho_{m}$ is mass concentration, $\mathrm{t} / \mathrm{m}^{3} ; v_{i}$ is inlet velocity, $\mathrm{m} / \mathrm{s}$.

2.2. Numerical Simulation Experimental Design. The numerical simulation experiment is designed by the commercial software Design Expert which is an experimental design software system. It can be used for statistical analysis, curve fitting, and mathematical modeling. Depending on the response surface to observe the law between the factors and responses, further optimal parameters of factors were obtained. Now the software has been extensively used in various types of multifactor test design and analysis.

Based on the central composite design of the response surface method according to the software Design Expert, the rotary quadratic combination design with three factors and five levels amounting to five groups of 20 test responses is constructed. The experimental design matrix comprised a full replication three-factor factorial design of 8 points, 8 axial points, and 6 center points. Experimental parameters and their levels are tabulated in Table 3.

The carbide slag sieving by standard sieve with 80 mesh per square inch at first and then stirring in a mixer for 30 minutes make the carbide slag particles sufficiently dispersed; 


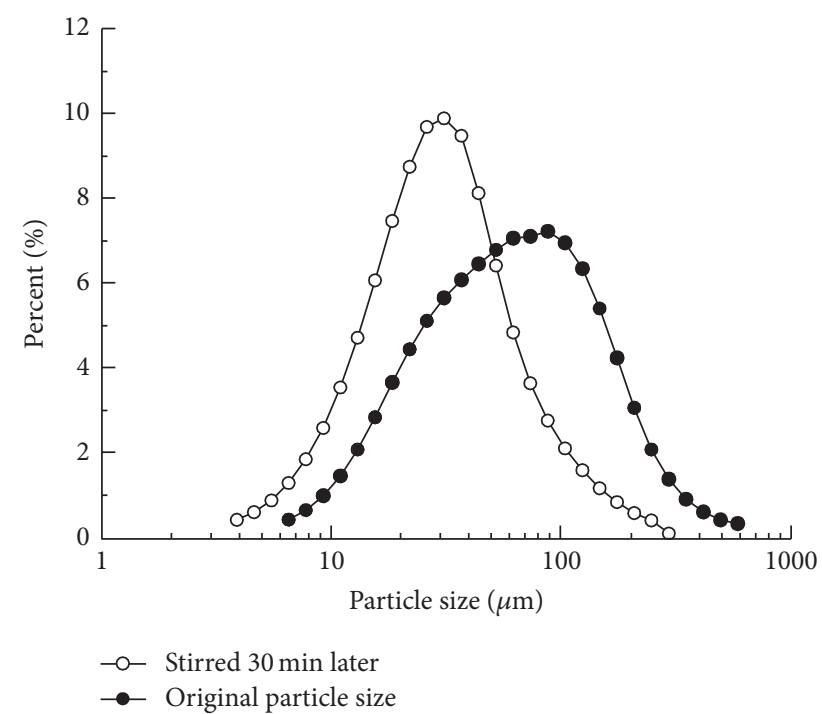

FIgURE 1: Particle size distribution of the carbide slag.

then using the Particle Size Analyzer-3500 analysis, the particle size distribution's result is shown in Figure 1.

Figure 1 shows that, in contrast with the original carbide slag, the particle size changed largely through stirring. The result significantly implied the dispersion effect of stir on agglomerated carbide slag. Because the larger particle separated more easily than a small particle in hydrocyclone, we use the smaller particle size (granularity: $3.18-36.82 \mu \mathrm{m}$ ) in analysis; this could realize that the small particle separated, simultaneously ensuring that the larger particle of impurities effectively separated.

Based on the input factors and their levels listed in Table 3, the numerical simulation experiments were conducted by the quadratic orthogonal rotation combination design and the experimental result is given in Table 4. Center points were used for getting a good estimate of experimental error. Because each group of simulation steady state usually has different time steps of iteration, and also the software itself has the error of calculation, the test response of six groups of center points acquired by adjusting the number of time steps of iteration could estimate the error of experimental simulation.

The experimental data acquired by the FLUENT software is disposed as follows.

(1) The experimental data acquired by the software is defined and described as follows:

$P_{i}$ : pressure of inlet, computed by Average of Surface Vertex Values, KPa

$P_{o}$ : pressure of overflow, computed by Average of Surface Vertex Values, KPa

$Q_{m o}$ : the slurry of mass flow overflow, $\mathrm{kg} / \mathrm{s}$

$Q_{m_{1} o}$ : the mass flow overflow of $\mathrm{Ca}(\mathrm{OH})_{2}, \mathrm{~kg} / \mathrm{s}$

$Q_{m_{2} o}$ : the mass flow overflow of FeSi, $\mathrm{kg} / \mathrm{s}$

$Q_{m_{1}}:$ the mass flow inlet of $\mathrm{Ca}(\mathrm{OH})_{2}, \mathrm{~kg} / \mathrm{s}$

$Q_{m_{2} i}$ : the mass flow inlet of $\mathrm{FeSi}, \mathrm{kg} / \mathrm{s}$

$Q_{m_{2} u}$ : the mass flow underflow of FeSi, kg/s
TABLE 4: The quadratic orthogonal rotation combination design and result.

\begin{tabular}{lcccccccc}
\hline Run & $A\left(x_{1}\right)$ & $\begin{array}{c}B\left(x_{2}\right) \\
\mathrm{m} / \mathrm{s}\end{array}$ & $\begin{array}{c}C\left(x_{3}\right) \\
\mu \mathrm{m}\end{array}$ & $\begin{array}{c}y_{1} \\
\mathrm{KPa}\end{array}$ & $\begin{array}{c}y_{2} \\
\%\end{array}$ & $\begin{array}{c}y_{3} \\
\%\end{array}$ & $\begin{array}{c}y_{4} \\
\%\end{array}$ & $\begin{array}{c}y_{5} \\
\%\end{array}$ \\
\hline 1 & 0.10 & 3.00 & 10.00 & 20.3 & 9.02 & 85.6 & 85.0 & 19.1 \\
2 & 0.30 & 3.00 & 10.00 & 22.6 & 27.80 & 85.6 & 85.8 & 18.2 \\
3 & 0.10 & 7.00 & 10.00 & 128.8 & 8.06 & 87.3 & 79.5 & 34.6 \\
4 & 0.30 & 7.00 & 10.00 & 142.4 & 26.06 & 87.2 & 82.5 & 31.4 \\
5 & 0.10 & 3.00 & 30.00 & 19.0 & 4.58 & 93.9 & 46.8 & 81.6 \\
6 & 0.30 & 3.00 & 30.00 & 4.8 & 30.05 & 85.0 & 49.8 & 50.3 \\
7 & 0.10 & 7.00 & 30.00 & 117.4 & 0.61 & 97.7 & 5.7 & 97.5 \\
8 & 0.30 & 7.00 & 30.00 & 123.1 & 18.31 & 97.4 & 56.3 & 91.5 \\
9 & 0.03 & 5.00 & 20.00 & 59.0 & 1.34 & 93.3 & 45.6 & 81.0 \\
10 & 0.37 & 5.00 & 20.00 & 66.3 & 28.64 & 91.7 & 71.6 & 63.4 \\
11 & 0.20 & 1.64 & 20.00 & 5.7 & 17.42 & 86.2 & 81.7 & 25.5 \\
12 & 0.20 & 8.36 & 20.00 & 180.1 & 8.46 & 96.1 & 40.6 & 90.6 \\
13 & 0.20 & 5.00 & 3.18 & 66.8 & 19.33 & 85.1 & 90.3 & 10.3 \\
14 & 0.20 & 5.00 & 36.82 & 55.2 & 3.71 & 98.6 & 18.2 & 97.7 \\
15 & 0.20 & 5.00 & 20.00 & 57.0 & 14.23 & 88.5 & 69.8 & 44.6 \\
16 & 0.20 & 5.00 & 20.00 & 58.2 & 12.79 & 89.3 & 67.5 & 51.0 \\
17 & 0.20 & 5.00 & 20.00 & 58.6 & 13.14 & 89.7 & 66.7 & 53.5 \\
18 & 0.20 & 5.00 & 20.00 & 61.4 & 12.86 & 92.4 & 62.0 & 70.6 \\
19 & 0.20 & 5.00 & 20.00 & 61.8 & 11.92 & 92.6 & 58.7 & 73.4 \\
20 & 0.20 & 5.00 & 20.00 & 61.1 & 11.87 & 92.4 & 56.8 & 73.7 \\
\hline
\end{tabular}

(2) The computational method related to the responses with factors is presented as follows:

$y_{1}:$ pressure drop, $\mathrm{KPa}$

$$
y_{1}=P_{i}-P_{o}
$$

$y_{2}$ : overflow concentration, $\%$

$$
y_{2}=100 \frac{Q_{m_{1} o}+Q_{m_{2} o}}{Q_{m o}}
$$

$y_{3}: \mathrm{Ca}(\mathrm{OH})_{2}$ overflow purity, $\%$

$$
y_{3}=100 \frac{Q_{m_{1} o}}{Q_{m_{1} o}+Q_{m_{2} o}}
$$

$y_{4}: \mathrm{Ca}(\mathrm{OH})_{2}$ separation efficiency, $\%$

$$
y_{4}=100 \frac{Q_{m_{1} o}}{Q_{m_{1} i}}
$$

$y_{5}:$ FeSi separation efficiency, \%

$$
y_{5}=100 \frac{Q_{m_{2} u}}{Q_{m_{2} i}} .
$$

\section{Results and Discussion}

\subsection{Pressure Drop}

3.1.1. Analysis of Variance (ANOVA). In order to investigate the fitness and significance of the model, ANOVA was 
TABLE 5: Variance analysis of factors to pressure drop.

\begin{tabular}{lccccc}
\hline Source & Sum of squares & df & Mean square & $F$ value & Prob $>F$ \\
\hline Model & 42521.82 & 10 & 4252.18 & 1028.54 & $<0.0001$ \\
$A$ & 28.85 & 1 & 28.85 & 6.98 & 0.0268 \\
$B$ & 15210.82 & 1 & 15210.82 & 3679.26 & $<0.0001$ \\
$C$ & 67.29 & 1 & 67.29 & 16.28 & 0.0030 \\
$A B$ & 120.45 & 1 & 120.45 & 29.14 & 0.0004 \\
$A C$ & 74.55 & 1 & 74.55 & 18.03 & 0.0022 \\
$B C$ & 16.80 & 1 & 16.80 & 4.06 & 0.0746 \\
$A^{2}$ & 11.04 & 1 & 11.04 & 2.67 & 0.1367 \\
$B^{2}$ & 1950.11 & 1 & 1950.11 & 471.70 & $<0.0001$ \\
$A^{2} B$ & 46.81 & 1 & 46.81 & 11.32 & 0.0083 \\
$A^{2} C$ & 25.50 & 1 & 25.50 & 6.17 & 0.0348 \\
Residual & 37.21 & 9 & 4.13 & & \\
Lack of fit & 17.20 & 4 & 4.30 & 1.07 & 0.4571 \\
Pure error & 20.01 & 5 & 4.00 & & \\
Cor total & 42559.03 & 19 & & & \\
\hline Ad $R$-Squred & & & & &
\end{tabular}

Adj $R$-Squared $=0.9982 ;$ Pred $R$-Squared $=0.9937$; Adeq Precision $=115.668$.

performed using the software Design Expert. ANOVA also shows the effects of single factors and interaction of factors on responses. Based on Table 4, the variance analysis of factors of pressure drop is given in Table 5.

The result from ANOVA shows the model is significant. The final obtained equation to predict the pressure drop in terms of the actual factors is expressed as follows:

$$
\begin{aligned}
y_{1}= & -5.292+97.651 x_{1}+2.075 x_{2}-0.482 x_{3} \\
& -55.768 x_{1} x_{2}+8.044 x_{1} x_{3}-0.072 x_{2} x_{3} \\
& -297.671 x_{1}{ }^{2}+2.894 x_{2}{ }^{2}+187.923 x_{1}{ }^{2} x_{2} \\
& -27.743 x_{1}{ }^{2} x_{3} .
\end{aligned}
$$

3.1.2. Analysis of Factors Influence. Based on " $F$ Value" and "Prob $>F$ " of Table 5, the single factors influence pressure drop in the order of $B, C, A$. The relationship between factors and response obtained by the software is shown in Figure 2.

From Figure 2(a), we can see that the pressure drop increases with the increasing of slurry concentration especially when the velocity inlet is at a high value, but it changed little at a low value. The phenomenon is mainly caused by slurry viscosity increasing when slurry concentration increases; because the pressure is proportional to the viscosity, the inlet pressure increases; simultaneously, the higher concentration improved the interaction between particles in internal fluid and induced more pressure loss, so the overflow pressure drops; then the pressure drop increases. Similarly, the pressure is proportional to the velocity; the higher velocity inlet improved the inlet pressure; although the overflow pressure could be also increased, it increased a little in contrast with inlet pressure; because the high velocity induced more pressure loss in internal fluid, there has more pressure drop at a high velocity inlet.
TABLE 6: Variance analysis of factors to overflow concentration.

\begin{tabular}{lccccc}
\hline Source & Sum of squares & df & Mean square & $F$ value & Prob $>F$ \\
\hline Model & 19.22 & 10 & 1.92 & 160.42 & $<0.0001$ \\
$A$ & 11.88 & 1 & 11.88 & 991.46 & $<0.0001$ \\
$B$ & 0.26 & 1 & 0.26 & 21.76 & 0.0012 \\
$C$ & 2.92 & 1 & 2.92 & 243.68 & $<0.0001$ \\
$A B$ & 0.31 & 1 & 0.31 & 25.76 & 0.0007 \\
$A C$ & 1.11 & 1 & 1.11 & 93.01 & $<0.0001$ \\
$B C$ & 0.68 & 1 & 0.68 & 56.96 & $<0.0001$ \\
$A^{2}$ & 0.74 & 1 & 0.74 & 62.17 & $<0.0001$ \\
$C^{2}$ & 0.19 & 1 & 0.19 & 16.26 & 0.0030 \\
$A B C$ & 0.27 & 1 & 0.27 & 22.70 & 0.0010 \\
$A^{2} B$ & 0.05 & 1 & 0.05 & 4.10 & 0.0736 \\
Residual & 0.11 & 9 & 0.01 & & \\
Lack of fit & 0.09 & 4 & 0.02 & 4.69 & 0.0603 \\
Pure error & 0.02 & 5 & 0.00 & & \\
Cor total & 19.32 & 19 & & & \\
\hline Ad & & & &
\end{tabular}

Adj $R$-Squared $=0.9982 ;$ Pred $R$-Squared $=0.9101$; Adeq Precision $=49.867$.

Figure 2(b) shows the pressure drop decreasing with the increasing of granularity. The phenomenon is mainly caused by the interaction between particles. At the same concentration, there would be fewer particles when the particle has a larger granularity; then the fewer interaction reduced the pressure loss in internal fluid; so overflow pressure increases; then the pressure drop decreases.

Figure 2(c) shows the pressure drop obviously increasing with the increasing of velocity inlet; this is also due to the inlet pressure increasing with the inlet velocity and the overflow pressure relatively decreasing due to the internal pressure loss. Simultaneously, the granularity also has a little effect on pressure drop, that is, the pressure drop decreasing with the increasing of granularity.

\subsection{Analysis of Overflow Concentration}

3.2.1. Analysis of Variance. Based on Table 4, the variance analysis of factors to overflow concentration is shown in Table 6.

The result from ANOVA shows the model is significant. The final obtained equation to predict the overflow concentration in terms of the actual factors is expressed as follows:

$$
\begin{aligned}
y_{2} & =\exp \left(1.170+3.050 x_{1}+0.114 x_{2}+0.091 x_{3}\right. \\
& +1.572 x_{1} x_{2}-0.088 x_{1} x_{3}-0.033 x_{2} x_{3}+7.804 x_{1}{ }^{2} \\
& \left.-0.001 x_{3}^{2}+0.092 x_{1} x_{2} x_{3}-6.085 x_{1}{ }^{2} x_{2}\right) .
\end{aligned}
$$

3.2.2. Analysis of Factors Influence. Based on "F Value" and "Prob $>F$ " of Table 6, the single factor influences the overflow concentration in the order of $A, C, B$. The relationship between factors and response obtained by the software is shown in Figure 3.

From Figure 3(a), we can see that the overflow concentration increases with the increasing of slurry concentration, but the velocity inlet is opposite. The phenomenon, mainly 


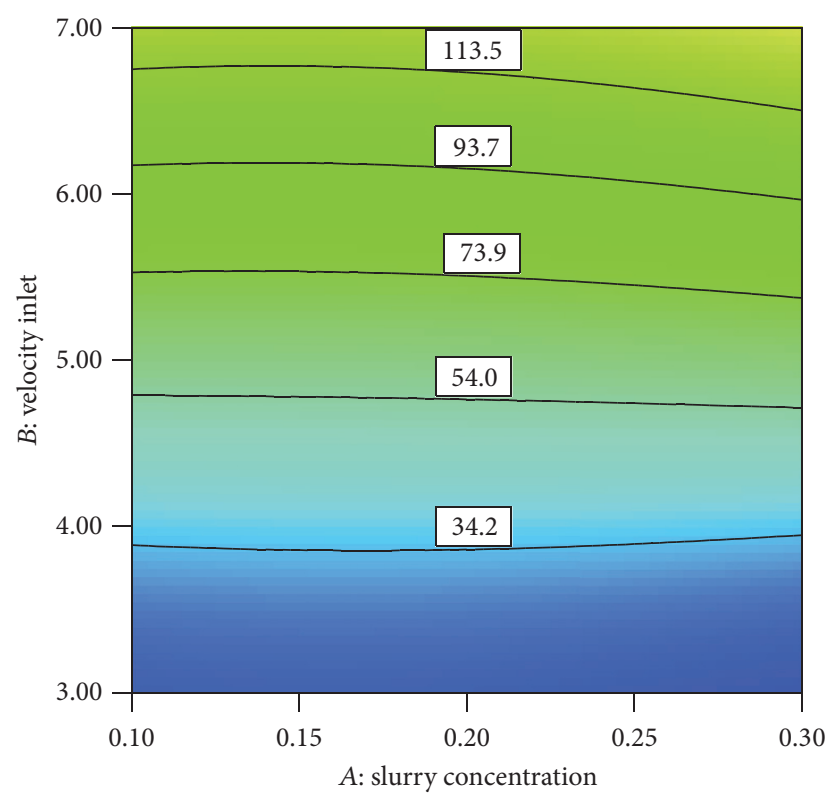

(a) $x_{3}=20.00$

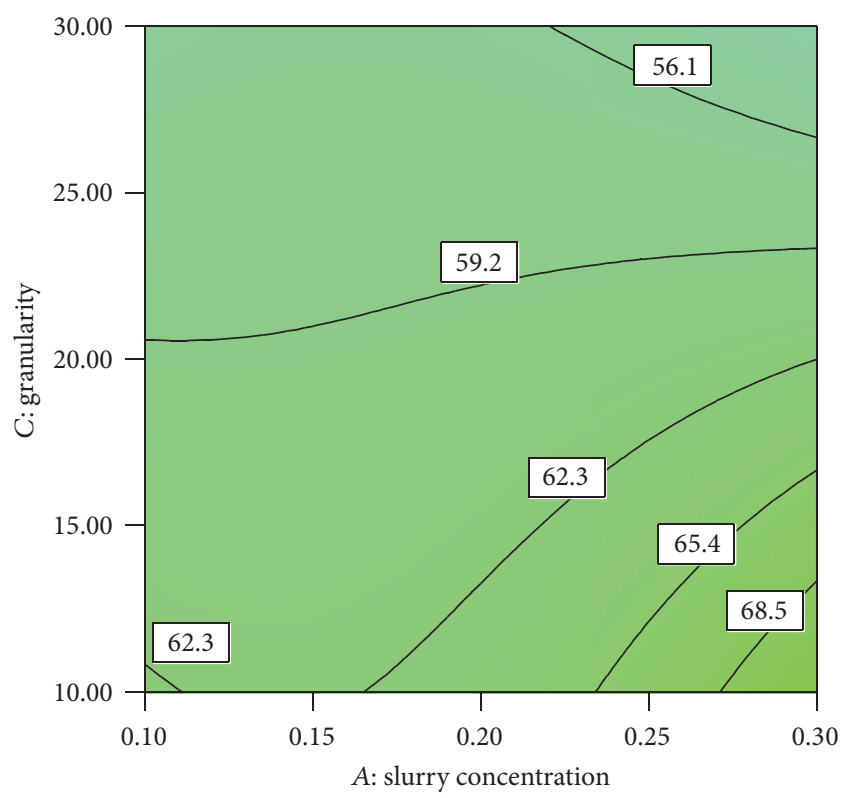

(b) $x_{2}=5.00$

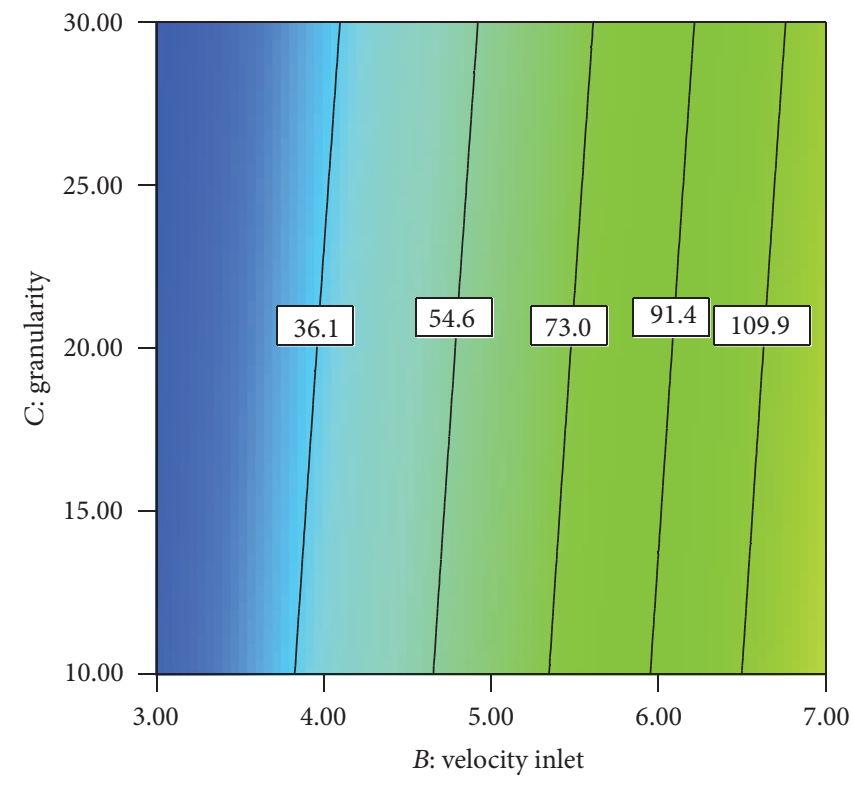

(c) $x_{1}=0.20$

FIGURE 2: Influence of factors on the pressure drop.

due to the slurry concentration, changed the amount of solid particles in liquid ratio; increasing concentration significantly improves the overflow and underflow concentration at the same time, and it has the largest influence on overflow concentration. Velocity inlet also has significant influence on overflow concentration; the higher the velocity, the bigger the centrifugal force of the particle obtained; then the particle tends more to wall of the hydrocyclone and separates to underflow, and then the overflow concentration decreases.

Figure 3(b) shows the overflow concentration decreasing with the granularity increasing, which is due to the fact that the bigger particle obtained the bigger centrifugal force, so the particle more easily separated to underflow, inducing the overflow concentration decrease.

From Figure 3(c), we can find that the overflow concentration has a little increase with the velocity increasing when the granularity is at a low value; the phenomenon could be due to the particle being too small; the small particle has a good flow characteristic with fluid, so it cannot separate well; simultaneously, the particle could have an impact on the wall of the hydrocyclone and rebound to overflow, inducing the overflow concentration increase. 


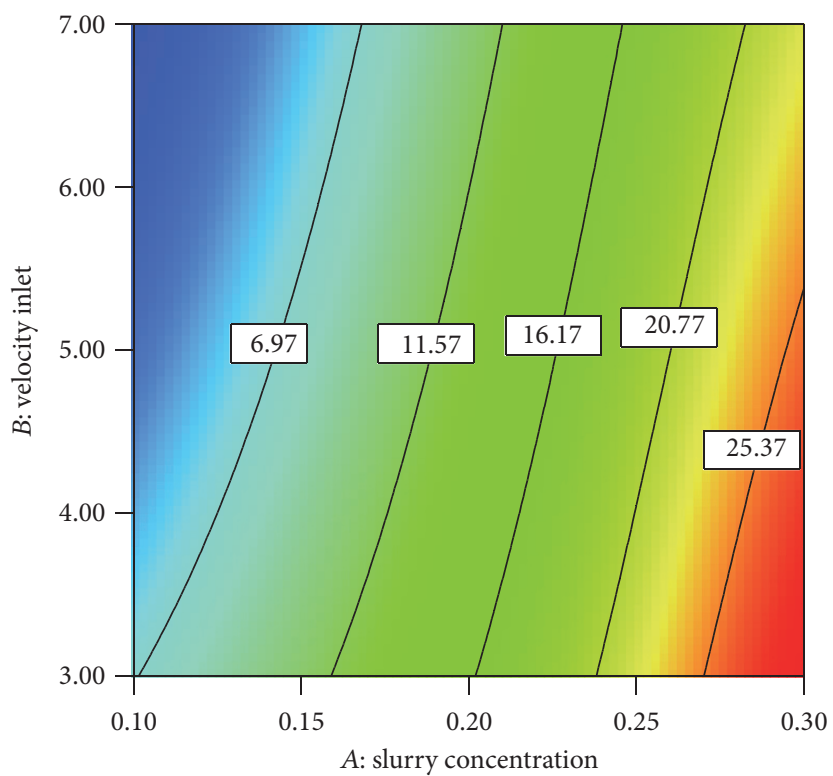

(a) $x_{3}=20.00$

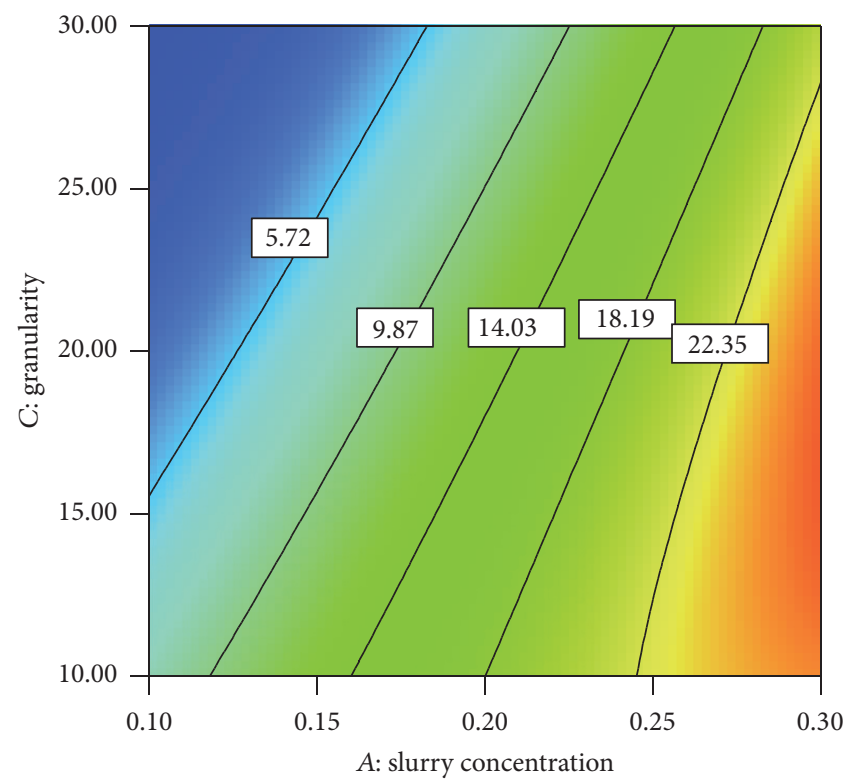

(b) $x_{2}=5.00$

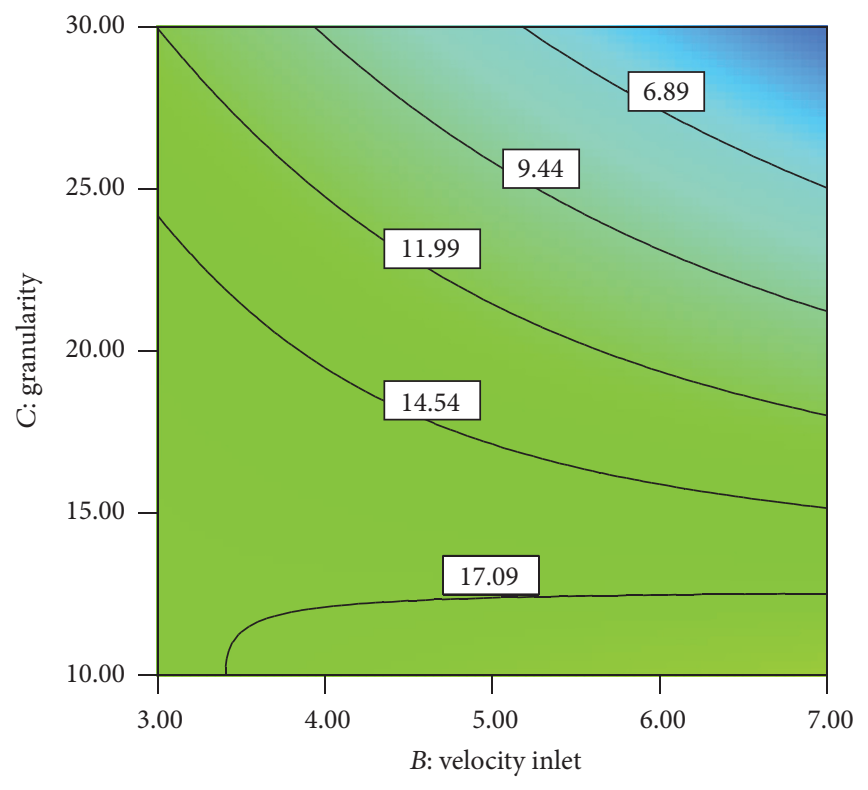

(c) $x_{1}=0.20$

FIGURE 3: Influence of factors on the overflow concentration.

\subsection{Analysis of Purity}

3.3.1. Analysis of Variance. Based on Table 4, the variance analysis of factors to overflow purity of $\mathrm{Ca}(\mathrm{OH})_{2}$ is given in Table 7.

The result from ANOVA shows the model is significant. The final obtained equation to predict the purity in terms of the actual factors is expressed as follows:

$$
\begin{aligned}
y_{3}= & 86.414-8.690 x_{1}-0.280 x_{2}-0.026 x_{3} \\
& +0.080 x_{2} x_{3} .
\end{aligned}
$$

3.3.2. Analysis of Factors Influence. Based on " $F$ Value" and "Prob $>F$ " of Table 7, the single factor influences the purity in the order of $C, B, A$, and the influence of $A$ is not significant. The relationship between factors and response obtained by the software is shown in Figure 4.

From Figure 4(a), we can see that the purity slightly decreases with the increasing of slurry concentration, and we find that the regularity of variation consists with factors $\left(x_{2}, x_{3}\right)$ change. The phenomenon could be due to the heightened interaction between particles with the slurry concentration increase; simultaneously, the higher concentration impedes the movement of the two solid phases at the 


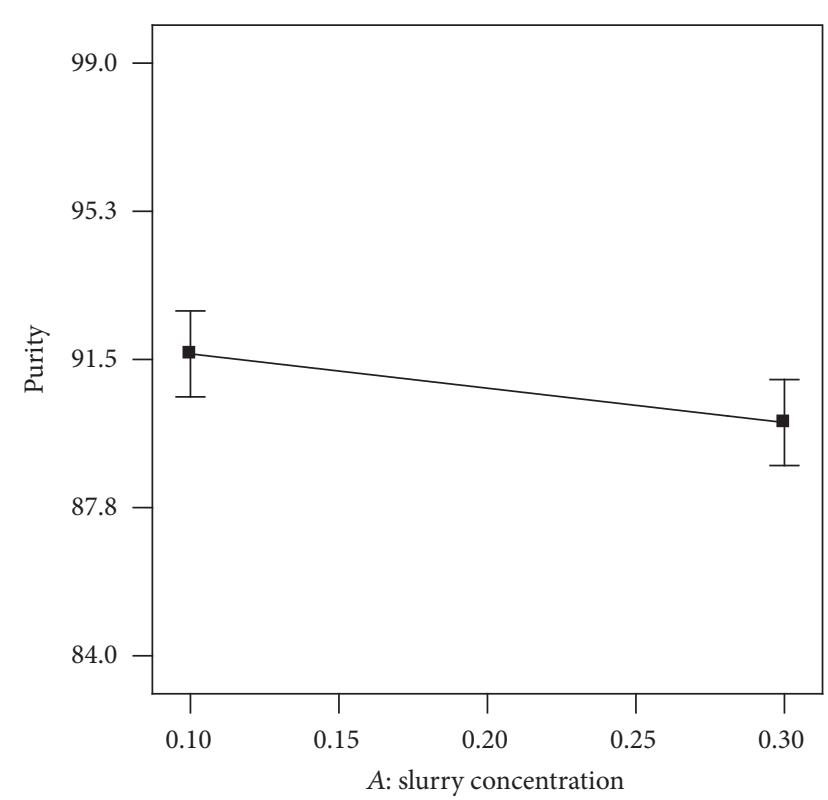

(a) $x_{2}=5.00, x_{3}=20.00$

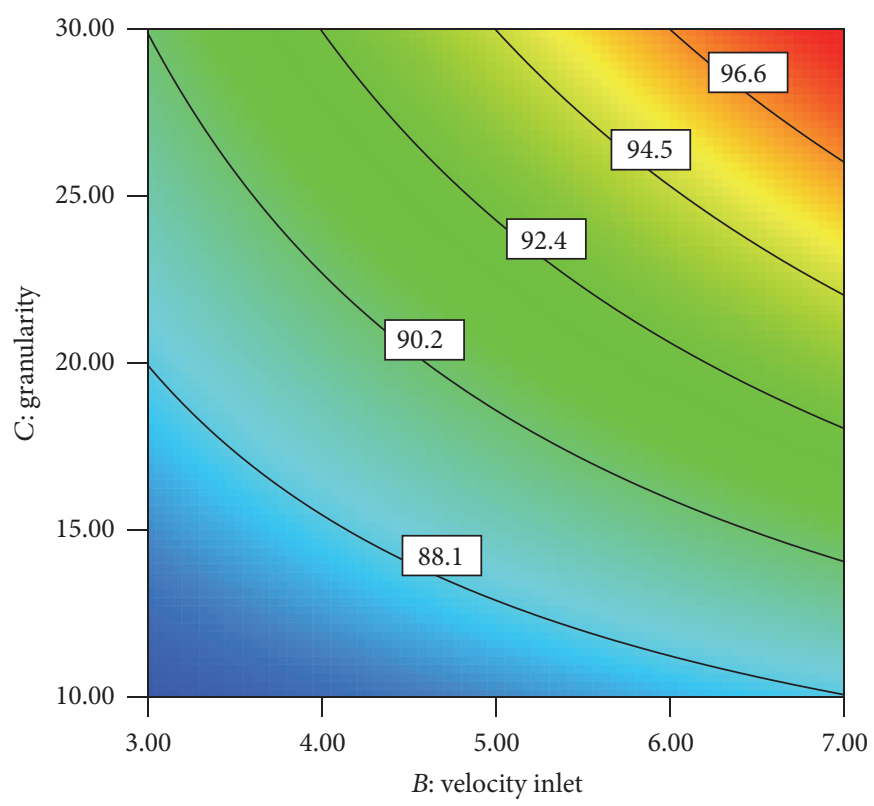

(b) $x_{1}=0.20$

FIGURE 4: Influence of factors on purity.

TABLE 7: Variance analysis of factors to purity.

\begin{tabular}{lccccc}
\hline Source & Sum of squares & df & Mean square & $F$ value & Prob $>$ F \\
\hline Model & 318.25 & 4 & 79.56 & 18.81 & $<0.0001$ \\
$A$ & 10.31 & 1 & 10.31 & 2.44 & 0.1393 \\
$B$ & 95.60 & 1 & 95.60 & 22.60 & 0.0003 \\
$C$ & 191.79 & 1 & 191.79 & 45.34 & $<0.0001$ \\
$B C$ & 20.55 & 1 & 20.55 & 4.86 & 0.0435 \\
Residual & 63.45 & 15 & 4.23 & & \\
Lack of fit & 46.34 & 10 & 4.63 & 1.35 & 0.3882 \\
Pure error & 17.11 & 5 & 3.42 & & \\
Cor total & 381.70 & 19 & & & \\
\hline
\end{tabular}

Adj $R$-Squared $=0.7894 ;$ Pred $R$-Squared $=0.6440 ;$ Adeq Precision $=14.749$.

radial direction of internal flow field of the hydrocyclone, decreasing the FeSi separation probability.

Figure 4(b) shows the purity increasing with the increase of velocity inlet and granularity. The phenomenon is mainly due to the difference of centrifugal force increasing between the two solid phases with the increase of velocity inlet and granularity. At the same condition, the higher density phase $(\mathrm{FeSi})$ tends more to move to outer vortex and separate to underflow and the lower density phase $\left(\mathrm{Ca}(\mathrm{OH})_{2}\right)$ tends more to move to internal vortex and separate to overflow, then inducing the purity increase.

\subsection{Analysis of $\mathrm{Ca}(\mathrm{OH})_{2}$ Separation Efficiency}

3.4.1. Analysis of Variance. Based on Table 4, the variance analysis of factors to $\mathrm{Ca}(\mathrm{OH})_{2}$ separation efficiency is given in Table 8.

The result from ANOVA shows the model is significant. The final obtained equation to predict the $\mathrm{Ca}(\mathrm{OH})_{2}$ separation
TABLE 8: Variance analysis of factors to $\mathrm{Ca}(\mathrm{OH})_{2}$ separation efficiency.

\begin{tabular}{lccccc}
\hline Source & Sum of squares & df & Mean square & $F$ value & Prob $>F$ \\
\hline Model & 57.44 & 8 & 7.18 & 73.21 & $<0.0001$ \\
$A$ & 5.20 & 1 & 5.20 & 52.97 & $<0.0001$ \\
$B$ & 5.88 & 1 & 5.88 & 59.99 & $<0.0001$ \\
$C$ & 33.88 & 1 & 33.88 & 345.50 & $<0.0001$ \\
$A B$ & 3.15 & 1 & 3.15 & 32.13 & 0.0001 \\
$A C$ & 3.28 & 1 & 3.28 & 33.42 & 0.0001 \\
$B C$ & 1.55 & 1 & 1.55 & 15.80 & 0.0022 \\
$C^{2}$ & 1.64 & 1 & 1.64 & 16.73 & 0.0018 \\
$A B C$ & 2.86 & 1 & 2.86 & 29.18 & 0.0002 \\
Residual & 1.08 & 11 & 0.10 & & \\
Lack of fit & 0.54 & 6 & 0.09 & 0.83 & 0.5910 \\
Pure error & 0.54 & 5 & 0.11 & & \\
Cor total & 58.52 & 19 & & & \\
\hline
\end{tabular}

Adj $R$-Squared $=0.9682 ;$ Pred $R$-Squared $=0.9335 ;$ Adeq Precision $=34.234$.

efficiency in terms of the actual factors is expressed as follows:

$$
\begin{aligned}
y_{4} & =\left(7.589+7.581 x_{1}+0.681 x_{2}+0.257 x_{3}\right. \\
& -2.843 x_{1} x_{2}-0.855 x_{1} x_{3}-0.082 x_{2} x_{3}-0.003 x_{3}{ }^{2} \\
& \left.+0.300 x_{1} x_{2} x_{3}\right)^{2} .
\end{aligned}
$$

3.4.2. Analysis of Factors Influence. Based on " $F$ Value" and "Prob $>F$ " of Table 8, the single factor influences $\mathrm{Ca}(\mathrm{OH})_{2}$ separation efficiency in the order of $C, B, A$. The relationship 


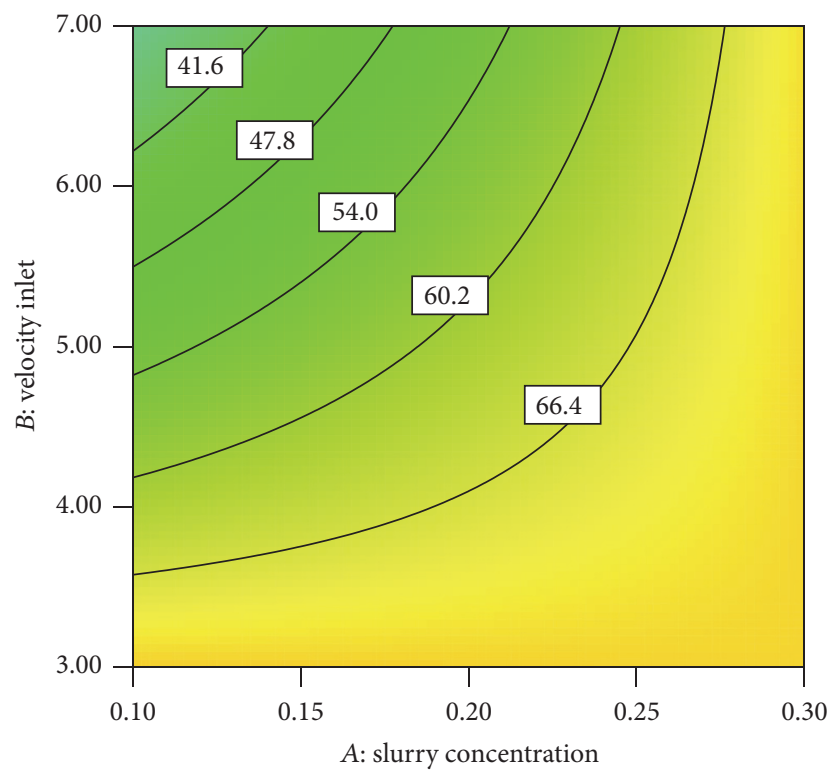

(a) $x_{3}=20.00$

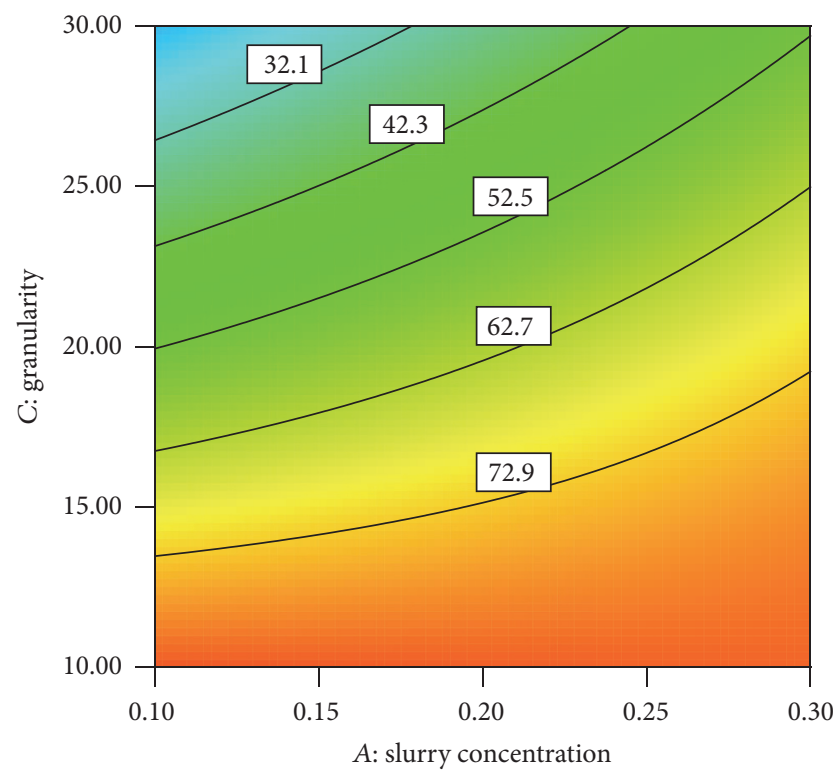

(b) $x_{2}=5.00$

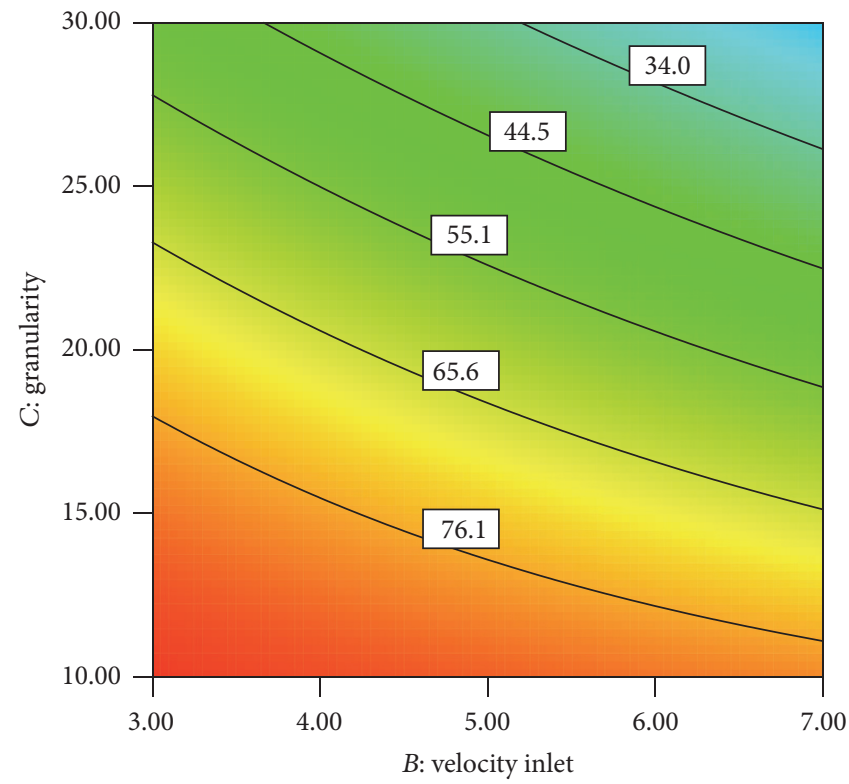

(c) $x_{1}=0.20$

Figure 5: Influence of factors on $\mathrm{Ca}(\mathrm{OH})_{2}$ separation efficiency.

between factors and response obtained by the software is shown in Figure 5.

Figure 5(a) shows that $\mathrm{Ca}(\mathrm{OH})_{2}$ separation efficiency increases with the increasing of slurry concentration, which is due to the fact that the solid phase of $\mathrm{Ca}(\mathrm{OH})_{2}$ has a lower density compared to $\mathrm{FeSi}$, so the $\mathrm{Ca}(\mathrm{OH})_{2}$ tends more to move into internal vertex and separate to overflow; simultaneously, the increasing of slurry concentration enhanced this effect and made more $\mathrm{Ca}(\mathrm{OH})_{2}$ separate to overflow; then, the $\mathrm{Ca}(\mathrm{OH})_{2}$ separation efficiency increased. We can also see that the $\mathrm{Ca}(\mathrm{OH})_{2}$ separation efficiency decreases with the increasing of velocity inlet, and this result is the same as in Figure 5(c); this is mainly caused by the effect of centrifugal sedimentation improved with the velocity inlet increasing, because the more solid phase of $\mathrm{Ca}(\mathrm{OH})_{2}$ moves into outer vortex and separates to underflow.

Figure 5(b) shows that the $\mathrm{Ca}(\mathrm{OH})_{2}$ separation efficiency decreases with the increasing of granularity, which is because the bigger granularity would get the stronger centrifugal sedimentation, so it would tend more to move into outer vortex and separate to underflow.

\subsection{Analysis of FeSi Separation Efficiency}

3.5.1. Analysis of Variance. Based on Table 4, the variance analysis of factors to FeSi separation efficiency is given in Table 9. 


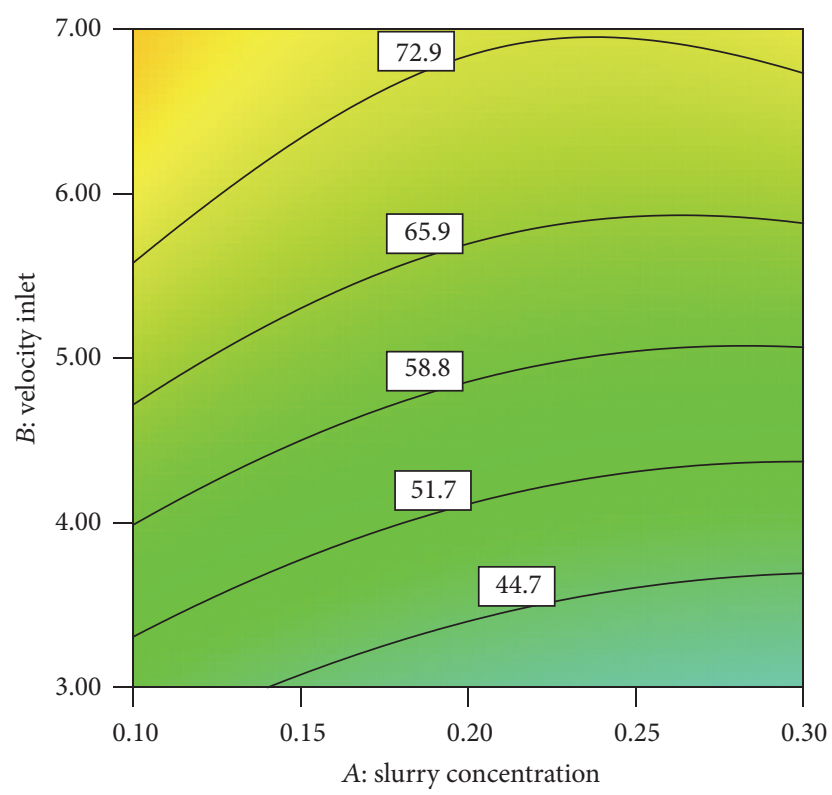

(a) $x_{3}=20.00$

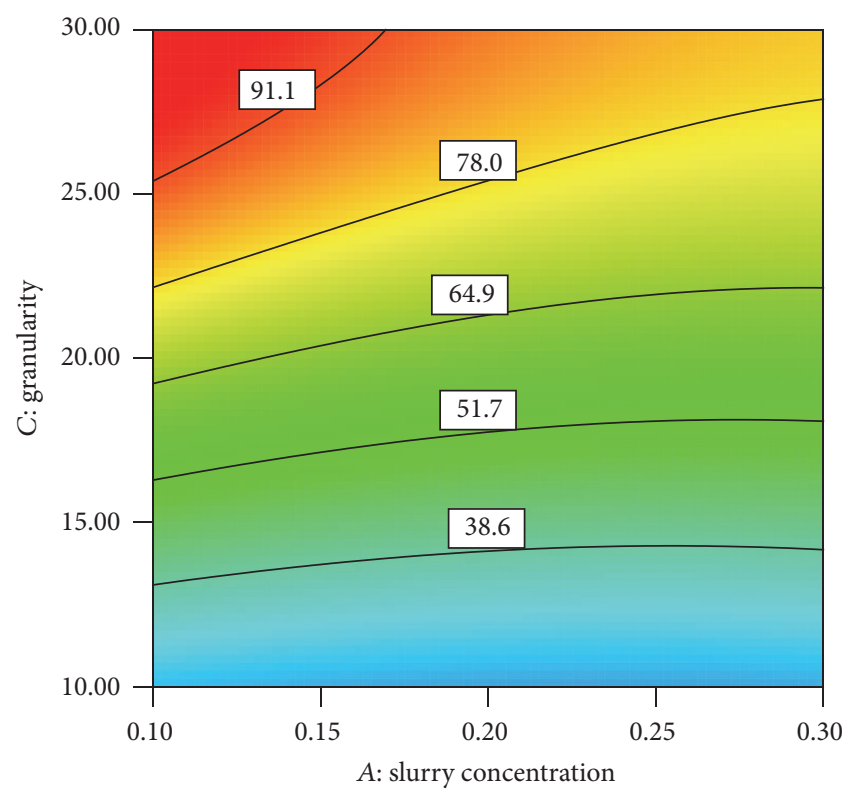

(b) $x_{2}=5.00$

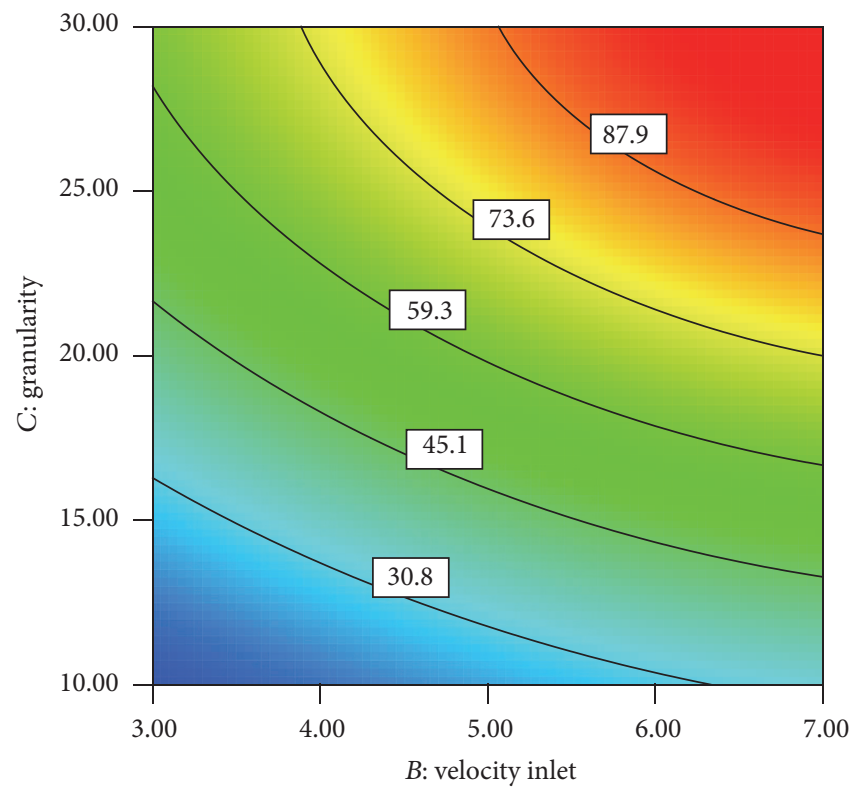

(c) $x_{1}=0.20$

Figure 6: Influence of factors on FeSi separation efficiency.

The result from ANOVA shows the model is significant. The final obtained equation to predict the FeSi separation efficiency in terms of the actual factors is expressed as follows:

$$
\begin{aligned}
y_{5} & =\exp \left(0.746-2.879 x_{1}+0.380 x_{2}+0.179 x_{3}\right. \\
& +0.230 x_{1} x_{2}-0.050 x_{1} x_{3}-0.002 x_{2} x_{3}+4.801 x_{1}{ }^{2} \\
& \left.-0.023 x_{2}{ }^{2}-0.002 x_{3}{ }^{2}\right) .
\end{aligned}
$$

3.5.2. Analysis of Factors Influence. Based on "F Value" and "Prob $>F$ " of Table 9, the single factor influences FeSi separation efficiency in the order of $C, B, A$, and the influence of $A$ is not significant. The relationship between factors and response obtained by the software is shown in Figure 6.

Figure 6(a) shows FeSi separation efficiency increasing with the slurry concentration increasing, which is due to the fact that the solid phase of FeSi has a higher density compared to $\mathrm{Ca}(\mathrm{OH})_{2}$, so the FeSi tends more to move into outer vertex and separate to underflow; simultaneously, the increasing of slurry concentration enhanced this effect and made more $\mathrm{FeSi}$ be separated to underflow; then the $\mathrm{FeSi}$ separation efficiency increased. We can also see that the FeSi separation efficiency increases with the increasing of velocity inlet, and this result is the same as in Figure 6(c); this is mainly caused by the effect of centrifugal sedimentation improved 
TABLE 9: Variance analysis of factors to FeSi separation efficiency.

\begin{tabular}{lccccc}
\hline Source & Sum of squares & df & Mean square & $F$ value & Prob $>F$ \\
\hline Model & 7.45 & 9 & 0.83 & 22.03 & $<0.0001$ \\
$A$ & 0.090 & 1 & 0.090 & 2.38 & 0.1537 \\
$B$ & 1.20 & 1 & 1.20 & 31.88 & 0.0002 \\
$C$ & 5.12 & 1 & 5.12 & 136.15 & $<0.0001$ \\
$A B$ & 0.017 & 1 & 0.017 & 0.45 & 0.5174 \\
$A C$ & 0.020 & 1 & 0.020 & 0.53 & 0.4817 \\
$B C$ & 0.016 & 1 & 0.016 & 0.43 & 0.5275 \\
$A^{2}$ & 0.033 & 1 & 0.033 & 0.88 & 0.3694 \\
$B^{2}$ & 0.12 & 1 & 0.12 & 3.32 & 0.0982 \\
$C^{2}$ & 0.83 & 1 & 0.83 & 22.16 & 0.0008 \\
Residual & 0.38 & 10 & 0.038 & & \\
Lack of fit & 0.14 & 5 & 0.028 & 0.59 & 0.7135 \\
Pure error & 0.24 & 5 & 0.047 & & \\
Cor total & 7.83 & 19 & & & \\
\hline
\end{tabular}

Adj $R$-Squared $=0.9088 ;$ Pred $R$-Squared $=0.8161 ;$ Adeq Precision $=17.306$.

with the velocity inlet increasing, which is due to the fact that the more solid phase of FeSi moves into outer vortex and separates to underflow. From Figure 6(a), we can also see that the FeSi separation efficiency tends to decrease as the slurry concentration and the velocity inlet are at the higher level. The phenomenon could be due to the fact that the higher level of the slurry concentration and the velocity inlet induced the internal flow field of hydrocyclone which changed to be unsteady, reducing the separation effect.

Figure 6(b) shows the FeSi separation efficiency increasing with the granularity, due to the fact that the bigger granularity would get the stronger centrifugal sedimentation, so it would tend more to move into the outer vortex and separate to underflow. This result tends to be more significant when the slurry concentration is at a lower level, because the lower concentration reduced the inhibition by interaction of particles, so the solid particles of FeSi would more easily move into the outer vortex and separate to underflow. From Figure 6(b), we can also see that the decreasing trend of the FeSi separation efficiency turns into being relatively slow with the slurry concentration increasing, and some even decrease. Similarly, this phenomenon could be also because the higher slurry concentration induced the interaction of particles improved, and the result impeded the diffuse particle into outer vortex and reduced the FeSi separated to underflow.

3.6. Multiobjective Optimization. The main objective of this study was to determine the optimized combination of factors with the required purity of $\mathrm{Ca}(\mathrm{OH})_{2}$ and suitable value of pressure drop and overflow concentration.

The first grade of limestone used in the production of calcium carbide demands that the $\mathrm{CaO}$ content reaches $88 \%$. According to this condition, the computational method of the demanded purity of $\mathrm{Ca}(\mathrm{OH})_{2}(\eta)$ is given by

$$
\begin{aligned}
\frac{(56 / 74) \eta}{1-(18 / 74) \eta} & =0.88 \Longrightarrow \\
\eta & =90.7 \% .
\end{aligned}
$$

TABLE 10: Constraints and results of numerical optimization.

\begin{tabular}{lcccc}
\hline Constraints & Goal & Lower & Upper & $\begin{array}{c}\text { Optimized } \\
\text { condition }\end{array}$ \\
\hline $\begin{array}{l}\text { Slurry } \\
\text { concentration }\end{array}$ & in range & 0.10 & 0.30 & 0.21 \\
$\begin{array}{l}\text { Velocity } \\
\text { inlet } / \mathrm{m} / \mathrm{s}\end{array}$ & in range & 3.00 & 7.00 & 6.22 \\
$\begin{array}{l}\text { Granularity } / \mu \mathrm{m} \\
\begin{array}{l}\text { Pressure } \\
\text { drop/KPa }\end{array}\end{array}$ & in range & 10.00 & 20.00 & 19.29 \\
$\begin{array}{l}\text { Overflow } \\
\text { concentration } / \%\end{array}$ & in range & 10.00 & 30.00 & 11.93 \\
\begin{tabular}{l} 
Purity/\% \\
\hline
\end{tabular} & In target & 92.0 & & 92.0 \\
\hline
\end{tabular}

TABLE 11: Results of simulation test.

\begin{tabular}{lccccc}
\hline Content & $y_{1} / \mathrm{KPa}$ & $y_{2} / \%$ & $y_{3} / \%$ & $y_{4} / \%$ & $y_{5} / \%$ \\
\hline Predicted results & 96.71 & 13.83 & 91.94 & 60.86 & 80.92 \\
Simulation results & 96.13 & 13.02 & 92.40 & 58.68 & 83.18 \\
Error/\% & 0.60 & 5.86 & 0.50 & 3.58 & 2.79 \\
\hline
\end{tabular}

We set the purity of $\mathrm{Ca}(\mathrm{OH})_{2}$ at $92.0 \%$, in order to meet the requirement and also it has some surplus to avoid the influence by uncertain factors.

Because $\mathrm{Ca}(\mathrm{OH})_{2}$ and impurities in carbide slag exist in state of cladding, the impurities cannot be separated completely in terms of theory. Even so, the process of separation can be proceeded, while the particles of carbide slag are small enough, which could be ensure that a large percent of impurities is separated, and then improve the purity of $\mathrm{Ca}(\mathrm{OH})_{2}$ in overflow. Based on multiobjective optimization, through adjusting the constraints, the range of granularity is set at $10.00-20.00 \mu \mathrm{m}$.

By setting the factors goal "in the range" and the responses goal to required range or value, the importance of factors in optimization has the same conditions and the numerical optimization can provide the optimized combination of factors. All the factors and responses of their lower and upper limits required for running numerical optimization are listed in Table 10, and the composition was optimized. The optimized conditions and results are shown in Table 10.

\subsection{Experiment Validation}

3.7.1. Simulation Test Validation. Based on optimized conditions (slurry concentration is 0.21 , velocity inlet is $6.22 \mathrm{~m} / \mathrm{s}$, and granularity is $19.29 \mu \mathrm{m}$ ), in contrast with the regression equations of responses $\left(y_{1}, y_{2}, y_{3}, y_{4}, y_{5}\right)$ and validation of the numerical simulation test, validation test results are shown in Table 11.

From Table 11, we can see that the predicted results are similar to the simulation results, except the overflow concentration exceeding 5\%; the other errors of predicted results to simulation results are all less than $5 \%$. The results indicate that the regression equations obtained by the method of aforementioned could acquire a good performance in predicting the numerical simulation result. 


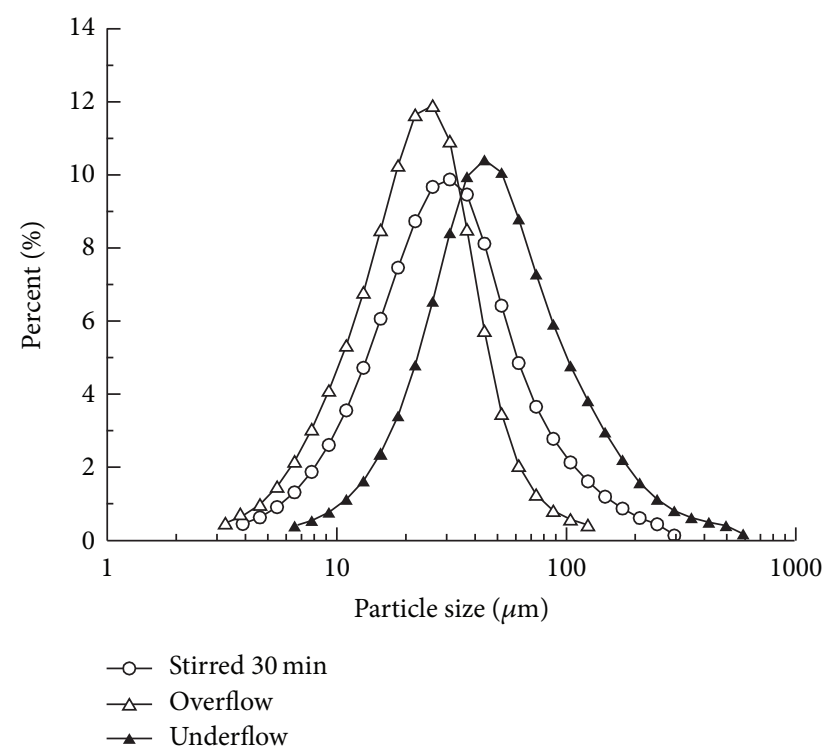

FIgURE 7: Particle size distribution of the separation result.

3.7.2. Separation Test Validation. Based on optimized conditions, the separation tests were proceeded. Using the Particle Size Analyzer-3500 analysis, the separation test result of particle size distribution is shown in Figure 7.

Figure 7 shows that, through the separation test, small particles more concentrated in overflow and larger particles more concentrated in underflow. The purity of $\mathrm{Ca}(\mathrm{OH})_{2}$ increased with the particle size of carbide slag decrease [25]; therefore, the purity of $\mathrm{Ca}(\mathrm{OH})_{2}$ of overflow product could increase.

From further analysis of the separation test, the results acquired were as follows:

(1) The pressure drop is $105 \mathrm{KPa}$, similar to the predicted result $96.71 \mathrm{KPa}$.

(2) The carbide slag overflow concentration is $14.7 \%$, similar to the predicted result $13.83 \%$.

(3) Because the separation efficiency of $\mathrm{Ca}(\mathrm{OH})_{2}$ and FeSi tested difficult, we use the carbide slag of overflow separation efficiency as the reference, and the value is $63 \%$, similar to the predicted result of $\mathrm{Ca}(\mathrm{OH})_{2}$ separation efficiency $60.86 \%$.

(4) The purity of $\mathrm{Ca}(\mathrm{OH})_{2}$ in original carbide slag, overflow product, and underflow product were tested by titration method, and the value is $86.67 \%, 94.86 \%$, $73.52 \%$, respectively.

Based on the separation test results, we can see that the predicted results are similar to the separation test results. The purity of $\mathrm{Ca}(\mathrm{OH})_{2}$ of overflow product is $94.86 \%$ greater than the simulation result $92.40 \%$ and predicted result $91.94 \%$; this result could be the density of FeSi as the impurity we set in simulation is less than the other impurities in carbide slag, so more impurities were separated into underflow; therefore, the purity of $\mathrm{Ca}(\mathrm{OH})_{2}$ of overflow product increased.

\section{Conclusions}

(1) The regression equations obtained by combined method (which is the method combined with the DOE method and CFD method) could acquire a good performance in predicting the numerical simulation result.

(2) The combined method could obtain more information in factors influence than traditional methods, especially on factors that have interaction and simultaneously have more efficiency.

(3) Although the particle size of the solid phases has a distribution character, the setting of the two phases with same granularity does not match the actual conditions, but if we use a reasonable method to improve the margin to avoid these influences, we can also acquire a good expectation in application engineering.

\section{Competing Interests}

The authors declare that there are no competing interests regarding the publication of this article.

\section{Acknowledgments}

The study was supported by Project no. 51264034 by National Natural Science Foundation of China and Project no. 2015ZRKXYQ04 supported by Basic Research for Application of Shihezi University.

\section{References}

[1] F. Liu, X. Wang, and J. Cao, "Effect of ultrasonic process on carbide slag activity and synthesized xonotlite," Physics Procedia, vol. 25, pp. 56-62, 2012.

[2] S. Zhang, X. Gong, Z. Wang, J. Cao, and Z. Guo, "Preparation of block $\mathrm{CaO}$ from carbide slag and its compressive strength improved by $\mathrm{H}_{3} \mathrm{PO}_{4}$," International Journal of Mineral Processing, vol. 129, pp. 6-11, 2014.

[3] H. Yang, J. Cao, Z. Wang, H. Chen, and X. Gong, "Discovery of impurities existing state in carbide slag by chemical dissociation," International Journal of Mineral Processing, vol. 130, pp. 66-73, 2014.

[4] S. U. Özgen, A. Yıldız, A. Çalışkan, and E. Sabah, "Modeling and optimization of hydrocyclone processing of low grade bentonites," Applied Clay Science, vol. 46, no. 3, pp. 305-313, 2009.

[5] S. Swain and S. Mohanty, "A 3-dimensional Eulerian-Eulerian CFD simulation of a hydrocyclone," Applied Mathematical Modelling, vol. 37, no. 5, pp. 2921-2932, 2013.

[6] S.-L. Gao, D.-Z. Wei, W.-G. Liu, L.-Q. Ma, T. Lu, and R.-Y. Zhang, "CFD numerical simulation of flow velocity characteristics of hydrocyclone," Transactions of Nonferrous Metals Society of China, vol. 21, no. 12, pp. 2783-2789, 2011.

[7] A. J. Lynch, T. C. Rao, and C. W. Bailey, "The influence of design and operating variables on the capacities of hydrocyclone classifiers," International Journal of Mineral Processing, vol. 2, no. 1, pp. 29-37, 1975. 
[8] L. R. Plitt, "Mathematical model of the hydrocyclone classifier," CIM Bulletin, vol. 69, no. 776, pp. 114-123, 1976.

[9] K. T. Hsieh and K. Rajamani, "Phenomenological model of the hydrocyclone: model development and verification for singlephase flow," International Journal of Mineral Processing, vol. 22, no. 1-4, pp. 223-237, 1988.

[10] W. Chen, N. Zydek, and F. Parma, "Evaluation of hydrocyclone models for practical applications," Chemical Engineering Journal, vol. 80, no. 1-3, pp. 295-303, 2000.

[11] L. Zhao, M. Jiang, and F. Li, "Experimental study on the separation performance of air-injected de-oil hydrocyclones," Chemical Engineering Research and Design, vol. 88, no. 5-6, pp. 772-778, 2010.

[12] Z. Jeirani, B. M. Jan, B. S. Ali, I. M. Noor, S. C. Hwa, and W. Saphanuchart, "The optimal mixture design of experiments: alternative method in optimizing the aqueous phase composition of a microemulsion," Chemometrics and Intelligent Laboratory Systems, vol. 112, pp. 1-7, 2012.

[13] G. Selvakumar, G. Sornalatha, S. Sarkar, and S. Mitra, "Experimental investigation and multi-objective optimization of wire electrical discharge machining (WEDM) of 5083 aluminum alloy," Transactions of Nonferrous Metals Society of China, vol. 24, no. 2, pp. 373-379, 2014.

[14] P. Dixit, R. Tiwari, A. K. Mukherjee, and P. K. Banerjee, "Application of response surface methodology for modeling and optimization of spiral separator for processing of iron ore slime," Powder Technology, vol. 275, pp. 105-112, 2015.

[15] B. Tang, Y. Xu, X. Song, Z. Sun, and J. Yu, "Numerical study on the relationship between high sharpness and configurations of the vortex finder of a hydrocyclone by central composite design," Chemical Engineering Journal, vol. 278, pp. 504-516, 2015.

[16] R. Arokiadass, K. Palaniradja, and N. Alagumoorthi, "Prediction and optimization of end milling process parameters of cast aluminium based MMC," Transactions of Nonferrous Metals Society of China, vol. 22, no. 7, pp. 1568-1574, 2012.

[17] D. O. Silva, L. G. M. Vieira, F. S. Lobato, and M. A. S. Barrozo, "Optimization of the design and performance of hydrocyclones by Differential Evolution technique," Chemical Engineering and Processing: Process Intensification, vol. 61, pp. 1-7, 2012.

[18] J. Averous and R. Fuentes, "Advances in the numerical simulation of hydrocyclone classification," Canadian Metallurgical Quarterly, vol. 36, no. 5, pp. 309-314, 1997.

[19] J. C. Cullivan, R. A. Williams, T. Dyakowski, and C. R. Cross, "New understanding of a hydrocyclone flow field and separation mechanism from computational fluid dynamics," Minerals Engineering, vol. 17, no. 5, pp. 651-660, 2004.

[20] J. A. Delgadillo and R. K. Rajamani, "A comparative study of three turbulence-closure models for the hydrocyclone problem," International Journal of Mineral Processing, vol. 77, no. 4, pp. 217-230, 2005.

[21] B. Wang and A. B. Yu, "Numerical study of particle-fluid flow in hydrocyclones with different body dimensions," Minerals Engineering, vol. 19, no. 10, pp. 1022-1033, 2006.

[22] K. U. Bhaskar, Y. R. Murthy, M. R. Raju, S. Tiwari, J. K. Srivastava, and N. Ramakrishnan, "CFD simulation and experimental validation studies on hydrocyclone," Minerals Engineering, vol. 20, no. 1, pp. 60-71, 2007.

[23] R. Safa and A. Soltani Goharrizi, "CFD simulation of an industrial hydrocyclone with Eulerian-Eulerian approach: A Case Study," International Journal of Mining Science and Technology, vol. 24, no. 5, pp. 643-648, 2014.
[24] P. He, M. Salcudean, and I. S. Gartshore, "A numerical simulation of hydrocyclones," Chemical Engineering Research and Design, vol. 77, no. 5, pp. 429-441, 1999.

[25] J. A. Feng, W. B. Wang, X. Q. Tang, Y. J. Qiu, and R. Ying, "Research on structural design of hydrocyclone for refining of carbide slag," Mining \& Processing Equipment, vol. 11, pp. 98-101, 2014 (Chinese). 


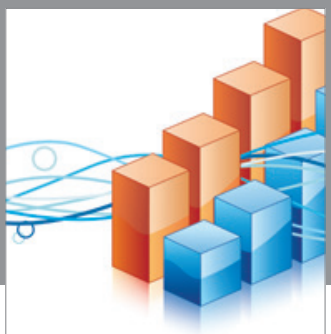

Advances in

Operations Research

vatem alat4

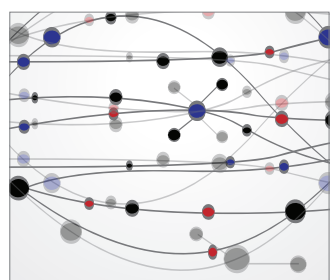

\section{The Scientific} World Journal
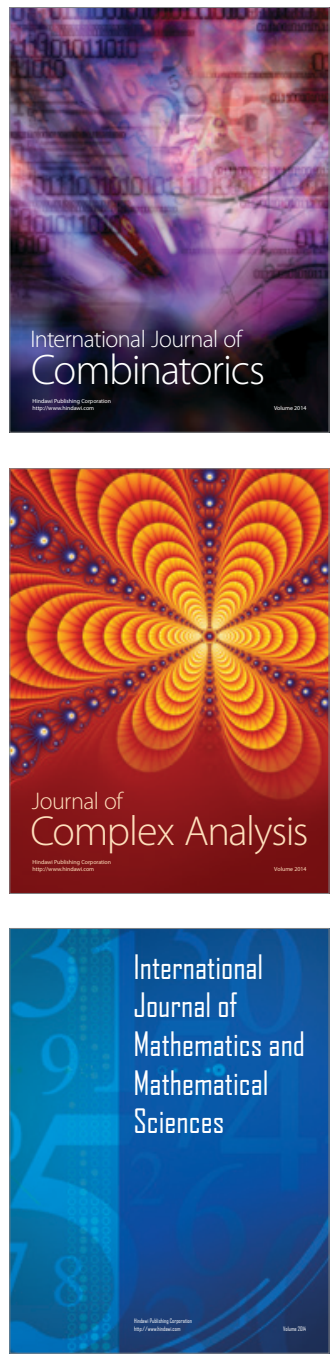
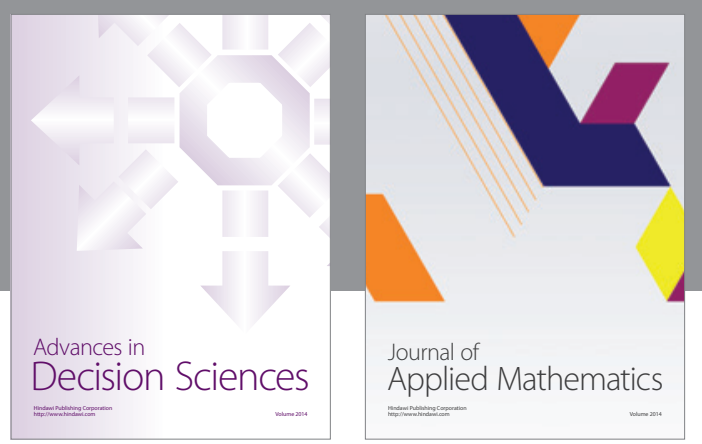

Algebra

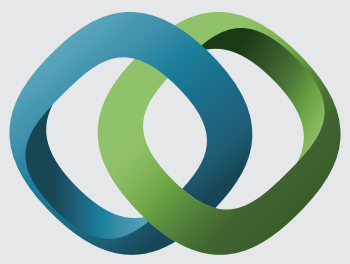

\section{Hindawi}

Submit your manuscripts at

http://www.hindawi.com
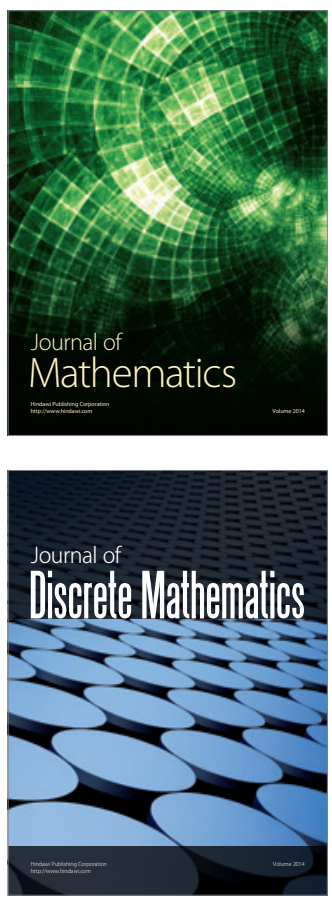

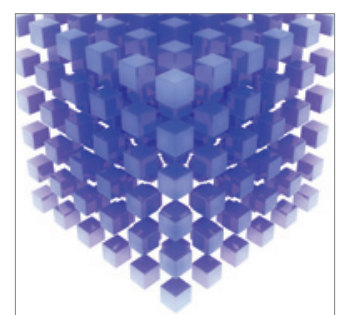

Mathematical Problems in Engineering
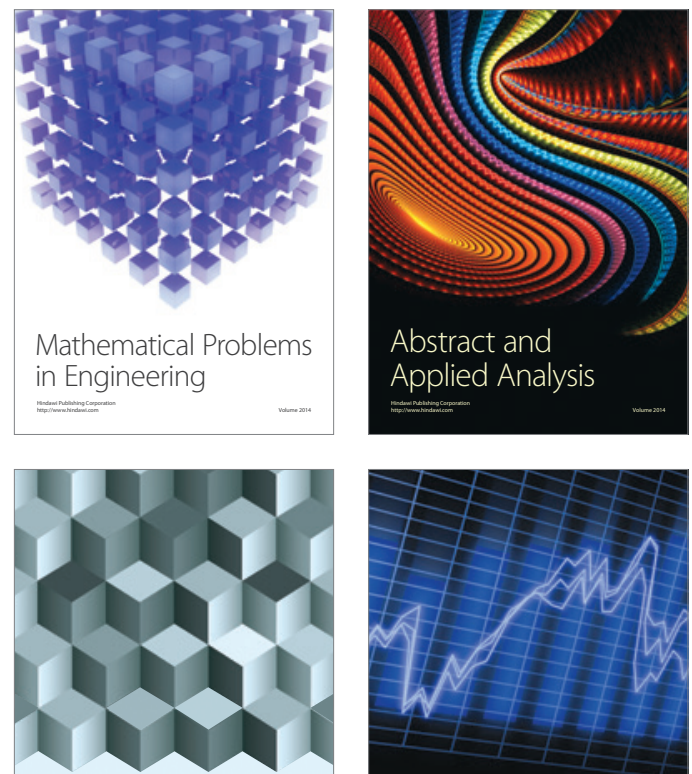

Journal of

Function Spaces

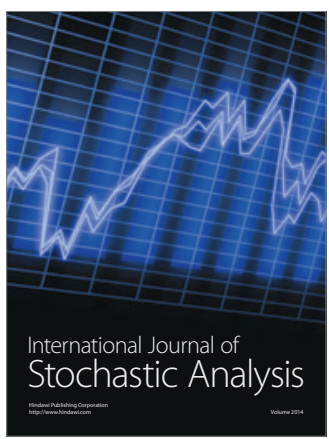

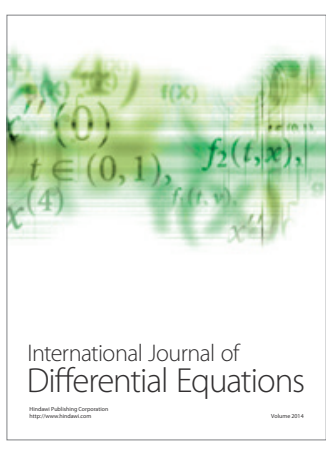
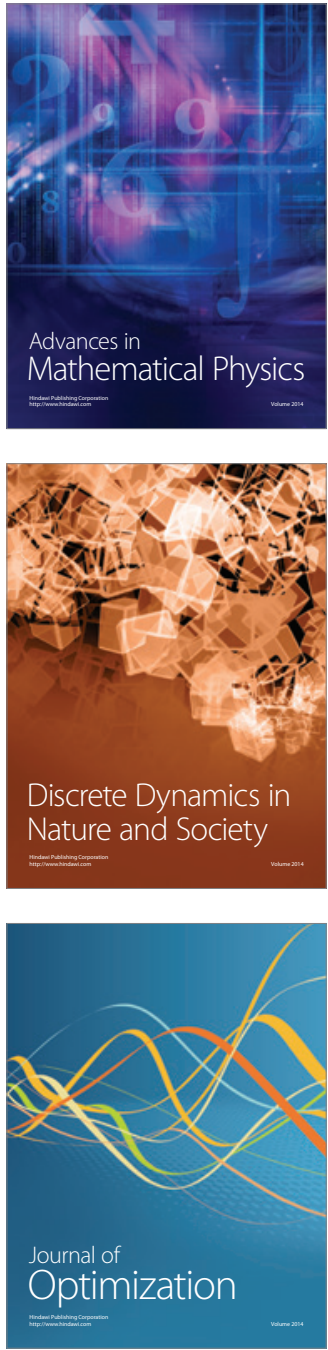